[23] J. J. Kuffner, K. Nishiwaki, S. Kagami, M. Inaba, and H. Inoue, "Motion planning for humanoid robots under obstacle and dynamic balance constraints," in Proc. IEEE Int. Conf. Robot. Autom., 2001, pp. 692-698.

[24] Z. Yao and K. Gupta, "Path planning with general end-effector constraints: Using task space to guide configuration space search," in Proc. IEEE/RSJ Int. Conf. Intell. Robots Syst., 2005, pp. 1875-1880.

[25] Z. Yao and K. Gupta, "Path planning with general end-effector constraints," Robot. Auton. Syst., vol. 55, no. 4, pp. 316-327, 2007.

[26] M. Stilman, J. Schamburek, J. Kuffner, and T. Asfour, "Manipulation planning among movable obstacles," in Proc. IEEE Int. Conf. Robot. Autom., 2007, pp. 3327-3332.

[27] L. Sciaviccio and B. Siciliano, Modeling and Control of Robot Manipulators. New York: McGraw-Hill, 1996.

[28] T. N. E. Greville, "Some applications of the pseudoinverse of a matrix," SIAM Rev., vol. 2, pp. 15-22, 1960.

[29] J. Yakey, S. LaValle, and L. E. Kavraki, "Randomized path planning for linkages with closed kinematic chains," IEEE Trans. Robot. Autom. vol. 17, no. 7, pp. 951-958, Dec. 2001.

[30] A. Atramentov and S. M. LaValle, "Efficient nearest neighbor searching for motion planning," in Proc. IEEE Int. Conf. Robot. Autom., 2002, pp. $1-6$.

[31] J. Kuffner and S. M. LaValle, "Rrt-connect: An efficient approach to single-query path planning," in Proc. Workshop Algorithmic Found. Robot., 2000, pp. 995-1001.

[32] P. R. Halmos, Finite-Dimensional Vector Spaces. New York: SpringerVerlag, 1974.

[33] B. Faverjon and P. Tournassoud, "A local based approach for path planning of manipulators with a high number of degrees of freedom," in Proc. IEEE Int. Conf. Robot. Autom., 1987, vol. 4, pp. 1152-1159.

[34] F. Kanehiro, F. Lamiraux, O. Kanoun, E. Yoshida, and J. P. Laumond, “A local collision avoidance method for non-strictly convex objects," in Proc. Robot.: Sci. Syst. Conf., 2008, pp. 1-8.

[35] T. Yoshikawa, "Manipulability of robotic mechanisms," Int. J. Robot. Res., vol. 4, pp. 3-9, 1985.

[36] H. Hanafusa, T. Yoshikawa, and Y. Nakamura, "Analaysis and control of articulated robot with redundancy," in Proc. IFAC, 8th Triennal World Congr., 1981, vol. 4, pp. 1927-1932.

[37] R. Boulic and P. Baerlocher, "Task-priority formulations for the kinematic control of highly redundant atriculated structures," in Proc. IEEE/RSJ Int. Conf. Intell. Robots Syst., 1998, pp. 323-329.

\section{Optimal Object Configurations to Minimize the Positioning Error in Visual Servoing}

\author{
Graziano Chesi, Senior Member, IEEE
}

\begin{abstract}
Image noise unavoidably affects the available image points that are used in visual-servoing schemes to steer a robot end-effector toward a desired location. As a consequence, letting the image points in the current view converge to those in the desired view does not ensure that the camera converges accurately to the desired location. This paper investigates the selection of object configurations to minimize the worst-case positioning error due to the presence of image noise. In particular, a strategy based on linear matrix inequalities (LMIs) and barrier functions is proposed to compute upper and lower bounds of this error for a given maximum error of the image points. This strategy can be applied to problems such as selecting an optimal subset of object points or determining an optimal position of an object in the scene. Some examples illustrate the use of the proposed strategy in such problems.
\end{abstract}

Index Terms-Image noise, positioning error, visual servoing.

\section{INTRODUCTION}

Visual servoing consists of steering a robot end-effector toward a desired location by exploiting, in a closed-loop fashion, the information provided by a vision system. Typically, the vision system is a camera mounted on the robot end-effector that observes the scene. The robot is controlled in order to make the projections of some object points in the current view converge to the projections of the same object points in the desired view, which have previously been recorded. Various methods have been proposed to accomplish this task, such as the pioneering image-based visual servoing (IBVS) [13] and position-based visual servoing (PBVS) [21]. Other methods use feedback errors, which are defined in both image and 3-D domains [18], partition of the degrees of freedoms [9], global motion plan via navigation functions [10], control invariant with respect to intrinsic parameters [17], switching control to ensure the visibility constraint [12], and path-planning to take various constraints into account [3]; see also [6] and references therein.

In all these methods, the robot control is terminated whenever the image points in the current view reach the corresponding ones in the desired view. However, this condition does not guarantee that the robot end-effector has accurately reached the sought desired location because the available image points in real visual-servo systems are unavoidably affected by image noise. This means that a positioning error unavoidably occurs.

This paper addresses this problem, in particular, investigating the worst-case robot positioning error that is introduced by image noise. Specifically, a strategy to compute upper and lower bounds of this error for a given maximum error of the image points is proposed. This strategy is based on the construction of suitable optimization problems with linear matrix inequalities (LMIs) and barrier functions that can readily be solved by using existing tools. Then, these bounds

Manuscript received July 28, 2009; revised November 30, 2009; accepted March 15, 2010. Date of publication April 12, 2010; date of current version June 9, 2010. This paper was recommended for publication by Associate Editor D. Kragic and Editor G. Oriolo upon evaluation of the reviewers' comments. This work was supported in part by the Research Grants Council of Hong Kong under Grant HKU711208E. This paper was presented in part at the International Conference on Robotics and Automation, Kobe, Japan, 2009.

The author is with the Department of Electrical and Electronic Engineering, University of Hong Kong, Hong Kong (e-mail: chesi@eee.hku.hk; http://www.eee.hku.hk/ chesi).

Digital Object Identifier 10.1109/TRO.2010.2046071 
are exploited in order to determine optimal object configurations that minimize this error. In particular, problems such as selecting an optimal subset of object points or determining an optimal position of an object in the scene can be considered. Some examples illustrate the use of the proposed strategy in such problems.

Before proceeding, it is worth mentioning that the effects of image noise in visual servoing have been investigated in the literature. In particular, Deng et al. [11] analyzed stability, robustness, sensitivity, and other characteristics of IBVS and PBVS. Then, Kyrki et al. [15] studied the propagation of image noise through pose estimation and control law in PBVS and 2-1/2-D visual servoing. Finally, Morel et al. [19] analyzed robustness with respect to calibration errors in terms of stability and tracking error.

The organization of this paper is as follows. Section II introduces the problem formulation. Section III describes the computation of upper and lower bounds and the selection of optimal object configurations. Section IV presents some illustrative examples. Last, Section V concludes the paper with some final remarks. This paper improves and extends our previous work [8].

\section{PRELIMINARIES}

\section{A. Notation and Problem Formulation}

The notations exploited in this paper are as follows.

1) $\mathbb{R}$ represents the space of real numbers.

2) $S O(3)$ is the set of rotation matrices in $\mathbb{R}^{3 \times 3}$.

3) $\mathbf{e}_{i}$ represents the $i$ th column of the $3 \times 3$ identity matrix.

4) $\|\mathbf{X}\|$ represents the 2 -norm of vector/matrix $\mathbf{X}$.

5) $\|\mathbf{X}\|_{\infty}$ represents the infinity norm of vector/matrix $\mathbf{X}$.

6) $\mathbf{X}^{T}$ represents the transpose of a vector/matrix $X$.

7) $\mathbf{X}>0$ (respectively, $\mathbf{X} \geq 0$ ) represents the positive-definite (respectively, positive-semidefinite) matrix $\mathbf{X}$.

8) w.r.t. means with respect to, and s.t. means subject to.

We denote the current camera frame with $F=(\mathbf{R}, \mathbf{t})$, where the rotation $\mathbf{R} \in S O(3)$ and translation $\mathbf{t} \in \mathbb{R}^{3}$ are defined w.r.t. the desired camera frame $F^{*}$. Let $\mathbf{q}_{i} \in \mathbb{R}^{3}$ be an object point expressed w.r.t. $F^{*}$. This point projects onto $F$ and $F^{*}$ at the points $\mathbf{p}_{i}=\left(p_{i, 1}, p_{i, 2}, 1\right)^{T}$ and $\mathbf{p}_{i}^{*}=\left(p_{i, 1}^{*}, p_{i, 2}^{*}, 1\right)^{T}$ defined by

$$
\begin{aligned}
d \mathbf{p}_{i} & =\mathbf{A} \mathbf{R}^{T}\left(\mathbf{q}_{i}-\mathbf{t}\right) \\
d^{*} \mathbf{p}_{i}^{*} & =\mathbf{A} \mathbf{q}_{i}
\end{aligned}
$$

where $d_{i}, d_{i}^{*} \in \mathbb{R}$ are the point depths, and $\mathbf{A} \in \mathbb{R}^{3 \times 3}$ is the uppertriangular matrix containing the camera intrinsic parameters. Let $\mathbf{q}_{1}, \ldots, \mathbf{q}_{N} \in \mathbb{R}^{3}$ be object points, and let $\mathbf{p}, \mathbf{p}^{*} \in \mathbb{R}^{2 N}$ be vectors containing their image projections $\mathbf{p}_{1}, \mathbf{p}_{1}^{*}, \ldots, \mathbf{p}_{N}, \mathbf{p}_{N}^{*}$ according to

$$
\begin{aligned}
\mathbf{p} & =\left(p_{1,1}, p_{1,2}, \ldots, p_{N, 1}, p_{N, 2}\right)^{T} \\
\mathbf{p}^{*} & =\left(p_{1,1}^{*}, p_{1,2}^{*}, \ldots, p_{N, 1}^{*}, p_{N, 2}^{*}\right)^{T}
\end{aligned}
$$

Visual servoing aims to position a robot end-effector by minimizing the distance between $\mathbf{p}$ and $\mathbf{p}^{*}$. Due to the presence of image noise, this error cannot be reduced to zero. Indeed, let us denote the estimates of $\mathbf{p}^{*}$ and $\mathbf{p}$ with $\hat{\mathbf{p}}^{*}$ and $\hat{\mathbf{p}}$ corrupted by image noise

$$
\left\{\begin{array}{l}
\hat{\mathbf{p}}=\mathbf{p}+\mathbf{n} \\
\hat{\mathbf{p}}^{*}=\mathbf{p}^{*}+\mathbf{n}^{*}
\end{array}\right.
$$

where $\mathbf{n}, \mathbf{n}^{*} \in \mathbb{R}^{2 N}$ contain image errors due to image noise. Let us suppose that $\mathbf{n}$ and $\mathbf{n}^{*}$ are bounded by

$$
\begin{aligned}
\|\mathbf{n}\|_{\infty} & \leq \eta \\
\left\|\mathbf{n}^{*}\right\|_{\infty} & \leq \eta
\end{aligned}
$$

for some $\eta \in \mathbb{R}$. Then, it follows that the goal condition

$$
\left\|\hat{\mathbf{p}}-\hat{\mathbf{p}}^{*}\right\|_{\infty} \leq \varepsilon
$$

for any threshold $\varepsilon \in \mathbb{R}$ guarantees only

$$
\left\|\mathbf{p}-\mathbf{p}^{*}\right\|_{\infty} \leq \varepsilon+2 \eta
$$

i.e., not even the choice $\varepsilon=0$ guarantees that $\mathbf{p}$ converges to $\mathbf{p}^{*}$.

Let us denote the maximum error on the image points as $\delta=\varepsilon+2 \eta$, and let us define the worst-case positioning error on the robot endeffector due to $\delta$ as

$$
\xi^{\#}=\sup _{\mathbf{R}, \mathbf{t}} \xi(\mathbf{R}, \mathbf{t}), \quad \text { s.t. }\left\|\mathbf{p}-\mathbf{p}^{*}\right\|_{\infty} \leq \delta
$$

where $\xi(\mathbf{R}, \mathbf{t})$ measures either the rotational or the translational error depending on the considered case, in particular

$$
\xi(\mathbf{R}, \mathbf{t})= \begin{cases}\theta, & \text { for rotational error } \\ \|\mathbf{t}\|, & \text { for translational error }\end{cases}
$$

where $\theta$ is the angle of $\mathbf{R}$ in the angle-axis representation, i.e., $\mathbf{R}=$ $e^{[\theta \mathbf{u}] \times}$ with $\theta \in[0, \pi]$, and $\|\mathbf{u}\|=1$.

The problem addressed in this paper is to select optimal object configurations, i.e., the points $\mathbf{q}_{1}, \ldots, \mathbf{q}_{N}$, in order to minimize $\xi^{\#}$. In particular, we consider

$$
\xi^{*}=\inf _{\mathbf{q}} \xi^{\#}, \quad \text { s.t. } \mathbf{q} \in \mathcal{Q} \cap \mathcal{V}
$$

where $\mathbf{q}=\left(\mathbf{q}_{1}^{T}, \ldots, \mathbf{q}_{N}^{T}\right)^{T}, \mathcal{Q} \subset \mathbb{R}^{3 N}$ is the set of allowed values for the vector $\mathbf{q}$, and $\mathcal{V}$ denotes the set of visible vectors $\mathbf{q}$ (which are the vectors $\mathbf{q}$ with all points $\mathbf{q}_{1}, \ldots, \mathbf{q}_{N}$ lying in the field of view of the camera).

It is worth remarking that image noise induces errors not only on the steady state but on the transient of the visual-servo system as well. The transient depends on the chosen control law, and hence, it is typically necessarily a case-by-case study [for PBVS, 2-1/2-D visual servoing, and IBVS, see [14] and [16]).

\section{B. Square Matricial Representation}

The approach proposed in this paper is based on the square matricial representation (SMR) of polynomials introduced in [7] to address a class of optimization problems over polynomials via LMI techniques. Specifically, a polynomial $y(\mathbf{x})$ of degree $2 m$ in the variable $\mathbf{x}=$ $\left(x_{1}, \ldots, x_{n}\right)^{T} \in \mathbb{R}^{n}$ can be expressed as

$$
y(\mathbf{x})=\mathbf{v}(\mathbf{x})^{T}(\mathbf{Y}+\mathbf{L}(\boldsymbol{\alpha})) \mathbf{v}(\mathbf{x})
$$


where $\mathbf{v}(\mathbf{x})$ is any vector containing a base for the polynomials of degree $m$ in $\mathbf{x}, \mathbf{Y}$ is any symmetric matrix such that

$$
y(\mathbf{x})=\mathbf{v}(\mathbf{x})^{T} \mathbf{Y} \mathbf{v}(\mathbf{x})
$$

$\boldsymbol{\alpha}$ is a vector of free parameters, and $\mathbf{L}(\boldsymbol{\alpha})$ is any linear parametrization of the set

$$
\mathcal{L}=\left\{\mathbf{L}=\mathbf{L}^{T}: \mathbf{v}(\mathbf{x})^{T} \mathbf{L} \mathbf{v}(\mathbf{x})=0 \quad \forall \mathbf{x}\right\} .
$$

The matrices $\mathbf{Y}$ and $\mathbf{Y}+\mathbf{L}(\boldsymbol{\alpha})$ are called SMR matrices of $y(\mathbf{x})$. The SMR finds application in various fields, such as computer vision [1], nonlinear systems [2], uncertain systems [4], and visual servoing [3] (for details and algorithms about the SMR, see [5]).

\section{Estimating AND Minimizing THE Positioning ERROR}

It turns out that computing $\xi^{\#}$ in (7) is a difficult problem due to the possible presence of local maxima, and hence, we present a strategy to estimate $\xi^{\#}$ via the computation of upper and lower bounds. This strategy is then exploited to select object configurations that minimize $\xi^{\#}$ and, hence, solve (9). Before proceeding, let us parametrize the rotation matrix $\mathbf{R}$ of the camera pose through quaternions [20] according to

$$
\mathbf{R}=\frac{\Omega(\mathbf{a})}{\|\mathbf{a}\|^{2}}
$$

where $\mathbf{a}=\left(a_{1}, \ldots, a_{4}\right)^{T} \in \mathbb{R}^{4}$, and $\boldsymbol{\Omega}: \mathbb{R}^{4} \rightarrow \mathbb{R}^{3 \times 3}$ is the homogeneous polynomial function of degree 2 given by

$$
\begin{aligned}
& \boldsymbol{\Omega}(\mathbf{a})=\left(\begin{array}{cc}
a_{1}^{2}-a_{2}^{2}-a_{3}^{2}+a_{4}^{2} & 2\left(a_{1} a_{2}-a_{3} a_{4}\right) \\
2\left(a_{1} a_{2}+a_{3} a_{4}\right) & -a_{1}^{2}+a_{2}^{2}-a_{3}^{2}+a_{4}^{2} \\
2\left(a_{1} a_{3}-a_{2} a_{4}\right) & 2\left(a_{2} a_{3}+a_{1} a_{4}\right)
\end{array}\right. \\
& 2\left(a_{1} a_{3}+a_{2} a_{4}\right) \\
& \left.2\left(a_{2} a_{3}-a_{1} a_{4}\right)\right) \text {. } \\
& \left.-a_{1}^{2}-a_{2}^{2}+a_{3}^{2}+a_{4}^{2}\right)
\end{aligned}
$$

For all nonzero vectors a, the right-hand side of (13) is an entry of $S O(3)$. Moreover, for any $\mathbf{R} \in S O(3)$, there exists an infinite number of nonzero vectors a such that (13) holds.

\section{A. Upper Bounds}

By eliminating the depths $d$ and $d^{*}$ in (1) and by taking into account the expression of $\mathbf{R}$ in (13), it follows that

$$
\mathbf{p}_{i}-\mathbf{p}_{i}^{*}=\mathbf{A} \frac{\boldsymbol{\Omega}(\mathbf{a})^{T} \mathbf{q}_{i}-\boldsymbol{\Omega}(\mathbf{a})^{T} \mathbf{t}}{\mathbf{e}_{3}^{T}\left(\boldsymbol{\Omega}(\mathbf{a})^{T} \mathbf{q}_{i}-\boldsymbol{\Omega}(\mathbf{a})^{T} \mathbf{t}\right)}-\mathbf{A} \frac{\mathbf{q}_{i}}{\mathbf{e}_{3}^{T} \mathbf{q}_{i}} .
$$

Let us define the new variable

$$
\mathbf{z}=-\frac{\boldsymbol{\Omega}(\mathbf{a})^{T}}{\|\mathbf{a}\|^{2}} \mathbf{t}
$$

and the set

$$
\begin{gathered}
\mathcal{I}=\{(i, j, k): i=1, \ldots, N, j=1,2, k=1,2\} \\
\bigcup\{(i, j, k): i=1, \ldots, N, j=3, k=1\} .
\end{gathered}
$$

For any triplet $(i, j, k) \in \mathcal{I}$, we define the polynomial

$$
h_{i, j, k}(\mathbf{a}, \mathbf{z})=(-1)^{k}\left(f_{i, 3} g_{i, j}(\mathbf{a}, \mathbf{z})-f_{i, j} g_{i, 3}(\mathbf{a}, \mathbf{z})\right)+\delta f_{i, 3} g_{i, 3}(\mathbf{a}, \mathbf{z})
$$

where $f_{i, j}$ and $g_{i, j}(\mathbf{a}, \mathbf{z})$ are given by

$$
\begin{aligned}
f_{i, j} & =\mathbf{e}_{j}^{T} \mathbf{A} \mathbf{q}_{i} \\
g_{i, j}(\mathbf{a}, \mathbf{z}) & =\mathbf{e}_{j}^{T} \mathbf{A}\left(\boldsymbol{\Omega}(\mathbf{a})^{T} \mathbf{q}_{i}+\|\mathbf{a}\|^{2} \mathbf{z}\right) .
\end{aligned}
$$

It can be verified that $\left\|\mathbf{p}-\mathbf{p}^{*}\right\|_{\infty} \leq \delta$ if and only if

$$
h_{i, j, k}(\mathbf{a}, \mathbf{z}) \geq 0 \quad \forall(i, j, k) \in \mathcal{I} .
$$

In fact, the polynomials $h_{i, j, k}(\mathbf{a}, \mathbf{z})$, with $j \in\{1,2\}$, allow one to suppose that each entry of the vector $\mathbf{p}_{i}-\mathbf{p}_{i}^{*}$ belongs to $[-\delta, \delta]$, while the polynomials $h_{i, j, k}(\mathbf{a}, \mathbf{z})$, with $j=3$, allow one to suppose that the object is in front of the cameras. Therefore, $\xi^{\#}$ can be rewritten as

$$
\xi^{\#}=\sup _{\mathbf{a}, \mathbf{z}} \xi(\mathbf{R}, \mathbf{t}), \quad \text { s.t. (20). }
$$

The next step involves adopting the SMR of polynomials described in Section II-B. To this end, let us introduce the polynomial

$$
b(\mathbf{a}, \mathbf{z})=b_{1}(\mathbf{a}, \mathbf{z})-\sum_{(i, j, k) \in \mathcal{I}} u_{i, j, k}(\mathbf{a}, \mathbf{z}) h_{i, j, k}(\mathbf{a}, \mathbf{z})
$$

where

$$
b_{1}(\mathbf{a}, \mathbf{z})= \begin{cases}\|\mathbf{a}\|^{2 l} \gamma-\|\mathbf{a}\|^{2(l-1)} \sum_{i=1}^{3} a_{i}^{2}, & \text { for rotational error } \\ \|\mathbf{a}\|^{2 l}\left(\gamma-\|\mathbf{z}\|^{2}\right), & \text { for translational error }\end{cases}
$$

where $l \geq 1$ is an integer, and $u_{i, j, k}(\mathbf{a}, \mathbf{z})$ are variable polynomials that are homogeneous in a of degree $2(l-1)$. The quantity $\gamma \in \mathbb{R}$ is a variable that encodes an upper bound of $\xi^{\#}$, as it will become clear in the sequel, in particular, whenever $b(\mathbf{a}, \mathbf{z})$ is nonnegative since this will imply that $b_{1}(\mathbf{a}, \mathbf{z})$ is nonnegative whenever (20) holds.

Let $\mathbf{v}_{b}(\mathbf{a}, \mathbf{z})$ be a vector containing any base for the polynomial $b(\mathbf{a}, \mathbf{z})$ (which is homogeneous in $\mathbf{a}$ of degree $2 l$ ), and let $\mathbf{v}_{u}(\mathbf{a}, \mathbf{z})$ be a similar vector for the polynomials $u_{i, j, k}(\mathbf{a}, \mathbf{z})$. Then, the polynomials $b(\mathbf{a}, \mathbf{z})$ and $u_{i, j, k}(\mathbf{a}, \mathbf{z})$ can be expressed through the SMR summarized in Section II-B, in particular, as

$$
\left\{\begin{array}{l}
b(\mathbf{a}, \mathbf{z})=\mathbf{v}_{b}(\mathbf{a}, \mathbf{z})^{T} \mathbf{B} \mathbf{v}_{b}(\mathbf{a}, \mathbf{z}) \\
u_{i, j, k}(\mathbf{a}, \mathbf{z})=\mathbf{v}_{u}(\mathbf{a}, \mathbf{z})^{T} \mathbf{U}_{i, j, k} \mathbf{v}_{u}(\mathbf{a}, \mathbf{z})
\end{array}\right.
$$

where $\mathbf{B}$ and $\mathbf{U}_{i, j, k}$ are any symmetric matrices satisfying (24) (for examples and algorithms about the construction of SMR matrices, see [5]). Let $\mathbf{N}(\boldsymbol{\alpha})$ be any linear parametrization of the set

$$
\mathcal{N}=\left\{\mathbf{N}=\mathbf{N}^{T}: \mathbf{v}_{b}(\mathbf{a}, \mathbf{z})^{T} \mathbf{N v}_{b}(\mathbf{a}, \mathbf{z})=0 \quad \forall \mathbf{a}, \mathbf{z}\right\}
$$

where $\boldsymbol{\alpha}$ is a free vector, and let us define

$$
\gamma^{*}=\inf _{\gamma, \boldsymbol{\alpha}, \mathbf{U}_{i, j, k}} \gamma, \quad \text { s.t. }\left\{\begin{array}{l}
\mathbf{B}+\mathbf{N}(\boldsymbol{\alpha}) \geq 0 \\
\mathbf{U}_{i, j, k} \geq 0 \quad \forall(i, j, k) \in \mathcal{I} .
\end{array}\right.
$$

The quantity $\gamma^{*}$ provides an upper bound of $\xi^{\#}$ via

$$
\xi_{+}^{\#}= \begin{cases}2 \arcsin \sqrt{\gamma^{*}}, & \text { for rotational error } \\ \sqrt{\gamma^{*}}, & \text { for translational error }\end{cases}
$$

since

$$
\xi_{+}^{\#} \geq \xi^{\#}
$$

In fact, by pre- and postmultiplying the first constraint in (26) by $\mathbf{v}_{b}(\mathbf{a}, \mathbf{z})^{T}$ and $\mathbf{v}_{b}(\mathbf{a}, \mathbf{z})$ and the second constraint by $\mathbf{v}_{u}(\mathbf{a}, \mathbf{z})^{T}$ and $\mathbf{v}_{u}(\mathbf{a}, \mathbf{z})$, it follows that

$$
\left.\begin{array}{r}
b(\mathbf{a}, \mathbf{z}) \geq 0 \\
u_{i, j, k}(\mathbf{a}, \mathbf{z}) \geq 0
\end{array}\right\} \quad \forall \mathbf{a}, \mathbf{z} \in \mathbb{R}^{3}
$$


and, hence

$$
b_{1}(\mathbf{a}, \mathbf{z}) \geq 0 \quad \forall \mathbf{a}, \mathbf{z} \in \mathbb{R}^{3}: h_{i, j, k}(\mathbf{a}, \mathbf{z}) \geq 0 \quad \forall(i, j, k) \in \mathcal{I} .
$$

Then, let us observe that the nonnegativity of $b_{1}(\mathbf{a}, \mathbf{z})$ implies, in the case of rotational error, that

$$
\gamma \geq \frac{\sum_{i=1}^{3} a_{i}^{2}}{\|\mathbf{a}\|^{2}}=\left(\sin \frac{\theta}{2}\right)^{2}
$$

while, in the case of translational error, that

$$
\gamma \geq\|\mathbf{z}\|^{2}=\|\mathbf{t}\|^{2}
$$

Hence, the original problem (21) has been transformed into the problem (26) by defining the polynomial $b(\mathbf{a}, \mathbf{z})$ through $u_{i, j, k}(\mathbf{a}, \mathbf{z})$, whose nonnegativity ensures that $\gamma$ encodes an upper bound of $\xi^{\#}$. Then, $\gamma$ is minimized under these nonnegativity constraints by using the SMR, in particular, imposing that the SMR matrices of these polynomials are positive semidefinite.

The minimization problem (26) is convex since the cost function is linear and the feasible set is convex (being the feasible set of LMIs) and can be solved by using dedicated MATLAB software such as the LMI toolbox or SeDuMi.

We observe that (27) is well-defined. In fact, for the rotational error, one has from (26) and (31) that $\gamma^{*}$ coincides with the maximum of the function $\|\mathbf{a}\|^{-2}\left(a_{1}^{2}+a_{2}^{2}+a_{3}^{2}\right)$ over some set of values for $\mathbf{a}$, and hence, $\gamma^{*} \in[0,1]$. Then, for the translational error, one has from (26) and (32) that $\gamma^{*}$ coincides with the maximum of the function $\|\mathbf{z}\|^{2}$ over some set of values for $\mathbf{z}$, and hence, $\gamma^{*} \geq 0$.

\section{B. Lower Bounds}

Let us consider now the computation of lower bounds of $\xi^{\#}$. Since $\|\mathbf{a}\|^{2}$ and $\boldsymbol{\Omega}(\mathbf{a})$ are homogeneous polynomials, it follows that there is no loss of generality in assuming that $\mathbf{a}$ is a unit-norm vector. The set of unit-norm vectors a can be parametrized via the function $\mathbf{s}: \mathbb{R}^{3} \rightarrow \mathbb{R}^{4}$ defined as

$$
\mathbf{s}(\mathbf{r})=\left(\begin{array}{c}
\sin r_{1} \sin r_{2} \sin r_{3} \\
\sin r_{1} \sin r_{2} \cos r_{3} \\
\sin r_{1} \cos r_{2} \\
\cos r_{1}
\end{array}\right)
$$

where $\mathbf{r}=\left(r_{1}, r_{2}, r_{3}\right)^{T}$ is a free vector. Hence, the positioning error $\xi^{\#}$ can be rewritten as

$$
\sup _{\mathbf{r}, \mathbf{t}} c_{1}(\mathbf{r}, \mathbf{t}) c_{2}(\mathbf{r}, \mathbf{t})
$$

where

$$
c_{1}(\mathbf{r}, \mathbf{t})= \begin{cases}1, & \text { if } h_{i, j, k}\left(\mathbf{s}(\mathbf{r}),-\boldsymbol{\Omega}(\mathbf{s}(\mathbf{r}))^{T} \mathbf{t}\right) \geq 0 \quad \forall(i, j, k) \in \mathcal{I} \\ 0, & \text { otherwise }\end{cases}
$$

and

$$
c_{2}(\mathbf{r}, \mathbf{t})=\xi(\boldsymbol{\Omega}(\mathbf{s}(\mathbf{r})), \mathbf{t}) .
$$

Let us observe that $c_{1}(\mathbf{r}, \mathbf{t})$ plays the role of a barrier function; in particular, it vanishes whenever the total image error is greater than $\delta$, and hence, it is not admissible. The optimization problem (34) can be tackled through any algorithm for nonlinear constrained optimization; among these algorithms, a good choice can be the Nelder-Mead simplex algorithm since it can handle nonsmooth optimization problems.
Let us observe that these algorithms may provide only a local maximum of (34); however, any found local maximum (that we denote as $\xi_{-}^{\#}$ ) provides directly a lower bound of $\xi^{\#}$, i.e.,

$$
\xi_{-}^{\#} \leq \xi^{\#} .
$$

It is also useful to observe that an admissible starting point in (34) is given by

$$
\mathbf{r}=(0,0,0)^{\prime}, \quad \mathbf{t}=(0,0,0)^{\prime}
$$

for all possible $\delta$, since this choice provides a total image error equal to zero.

\section{Optimal Object Configurations}

The upper and lower bounds $\xi_{+}^{\#}, \xi_{-}^{\#}$ can be used to bound the solution of (9) and, hence, to select an object configuration minimizing the worst-case positioning error $\xi^{\#}$. This can be done by defining

$$
\begin{array}{ll}
\xi_{+}^{*}=\inf _{\mathbf{q}} \xi_{+}^{\#}, & \text { s.t. } \mathbf{q} \in \mathcal{Q} \cap \mathcal{V} \\
\xi_{-}^{*}=\inf _{\mathbf{q}} \xi_{-}^{\#}, & \text { s.t. } \mathbf{q} \in \mathcal{Q} \cap \mathcal{V} .
\end{array}
$$

It follows that $\xi_{+}^{*}, \xi_{-}^{*}$ are bounds of $\xi^{\#}$; indeed, from (9), (28), and (37), one has

$$
\xi_{-}^{*} \leq \xi^{*} \leq \xi_{+}^{*} .
$$

Solving (39) can be difficult depending on the domain set of $q$. In general, one can try to simplify the problem by selecting a set $\mathcal{Q}$ with simple shape, such as a box. Hereafter, we discuss the solution of (39) in two typical cases.

As first case, let us observe that the scene often offers a number of object points that is larger than the one required by the visualservoing control law. However, not all object points can be considered, for instance, because this would not allow real-time applications and would make it more difficult to fulfill the visibility constraint during the camera motion. Hence, one has to select a subset of these object points to use, and the problem is to select the subset that minimizes the worst-case positioning error $\xi^{\#}$ in (7). This is equivalent to solving (9), where the set $\mathcal{Q}$ is a finite set given by the $N$-tuples (without ordering) that can be chosen with the $N_{p}$ visible object points present in the scene. This case can be addressed by evaluating the bounds $\xi_{+}^{\#}, \xi_{-}^{\#}$ in (28) and (37) for each of this tuple (see also Section IV-A).

The second case we consider is the determination of an optimal position of an object in the scene. Indeed, the scene often offers the possibility of freely locating an object within a certain region, which means that a group of object points can be chosen in order to minimize the worst-case positioning error $\xi^{\#}$ in (7). Specifically, the problem amounts to solving (9), where the set $\mathcal{Q}$ is described by the set of possible object points that can be obtained by letting the object move within its allowed region of the scene. The dimension of $\mathcal{Q}$ is given by the degrees of freedom of the object, which can be used to directly parametrize $\mathcal{Q}$. This case can be addressed by minimizing $\xi_{+}^{\#}, \xi_{-}^{\#}$ in (28) and (37) w.r.t. these degrees of freedom. In the specific case of $\xi_{+}^{\#}$, another possible way is to iterate between the variables of (28) and these degrees of freedom, since $\xi_{+}^{\#}$ is defined as a minimum (see also Section IV-B).

\section{EXAMPLES}

In this section, we present two illustrative examples of the investigation propose in this paper. The maximum error of the image points is $\delta=2$ pixels. For the camera intrinsic parameters, the focal lengths are 500 pixels, the aspect ratio is 0 , and the coordinates of the principal point are $(320,400)$ pixels. 


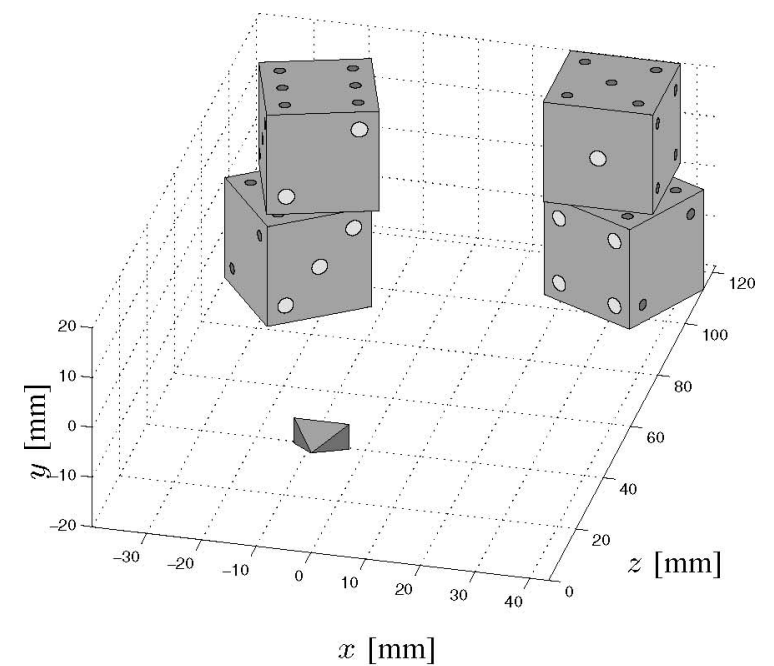

(a)

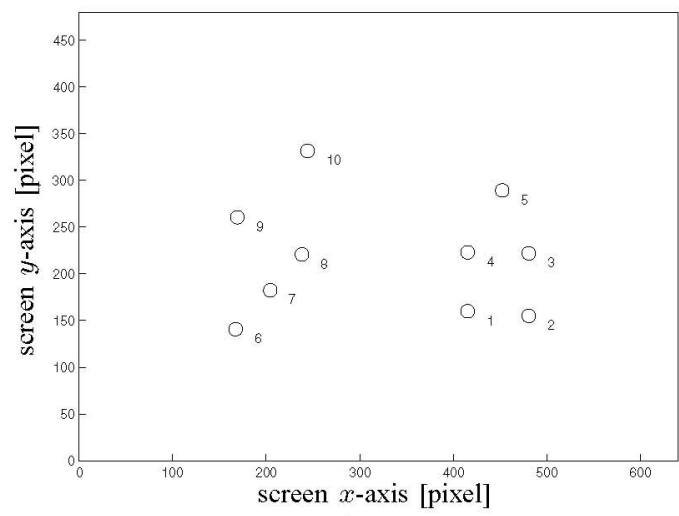

(b)

Fig. 1. Example 1. (a) Scene. (b) Camera view.

Before proceeding, it is worth mentioning that one could think of the estimation of the positioning error by calculating the camera pose corresponding to the image points corrupted with admissible values of the image error, for example, $\pm \delta$ on each coordinate. However, since the degrees of freedom of the camera pose are typically less than the number of constraints imposed by the considered image points, it follows that no camera pose fits exactly such image points, which means that an estimation error should be introduced.

\section{A. Example 1}

Let us consider the situation shown in Fig. 1(a), where a camera is observing $N_{p}=10$ object points (i.e., the center of the dots of four dices), and the problem is to determine the $N=8$ object points among these 10 that minimize the worst-case translational error. Fig. 1(b) shows the dots in the camera view, each of them with an assigned reference number. Hence, the target is to solve (9) where the set $\mathcal{Q}$ is the set of 8-tuples (without ordering) that can be chosen with the 10 object points present in the scene. To this end, we evaluate the bounds $\xi_{+}^{\#}, \xi_{-}^{\#}$ in (28) and (37) for each of this tuple, as described in Section III. Fig. 2 shows these bounds and their average versus the tuple number (the tuple number is sorted to obtain a nondecreasing average error). As we can see, the worst-case positioning error can significantly vary depending on the object points that are chosen. In particular, the tuple

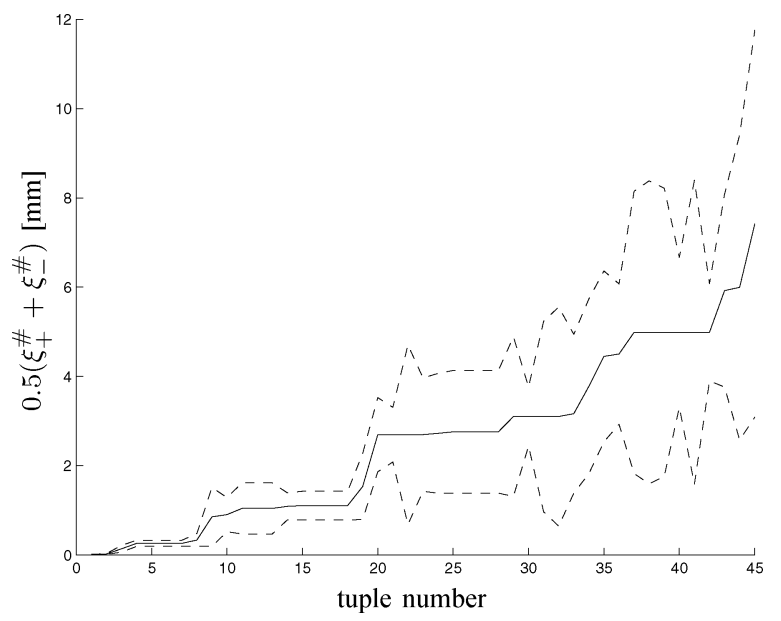

Fig. 2. Example 1. Bounds $\xi_{+}^{\#}, \xi_{-}^{\#}$ for the (dashed line) translational error and (solid line) their average versus the tuple number.

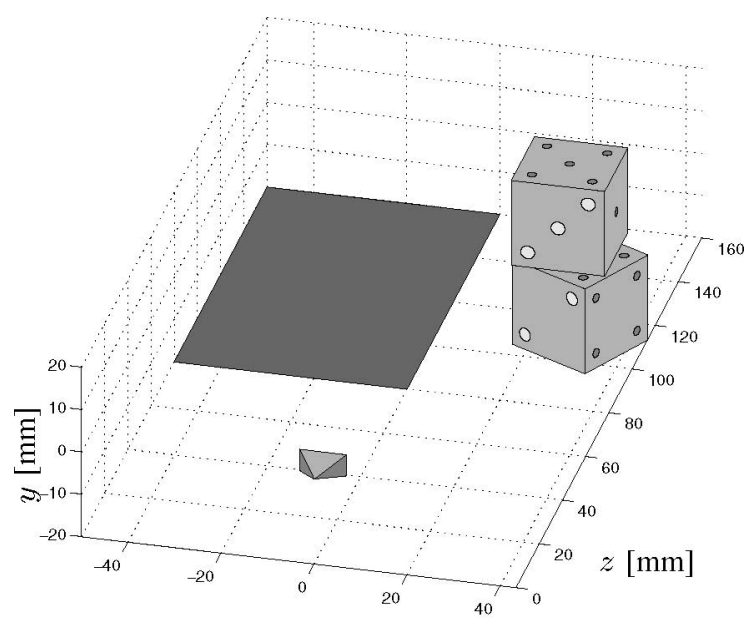

$x[\mathrm{~mm}]$

Fig. 3. Example 2. Scene with allowed region for the third dice.

of object points corresponding to the minimum of the curve in Fig. 2 is given by $(1,2,3,4,5,7,8,10)$.

\section{B. Example 2}

Let us consider the situation shown in Fig. 3, where a camera is observing five object points (i.e., the center of the dots of two dice), and the problem is to determine the $x-z$ position in the dashed area of the horizontal plane of a third dice with visible four-side (which is supposed parallel to the image plane) in order to minimize the worstcase translational error. Hence, the target is to solve (9) where the set $\mathcal{Q}$ is described by the set of possible $N=9$ object points that can be obtained by considering the two dices already present and letting the third die move within the dashed area. To this end, we minimize the average of the bounds $\xi_{+}^{\#}, \xi_{-}^{\#}$ in (28) and (37) via the Nelder-Mead simplex algorithm using the center of the dashed area as starting point, and we obtain that the optimal position for the third die is the topleft corner of the dashed area (the average of the bounds $\xi_{+}^{\#}, \xi_{-}^{\#}$ at this location is $2.07 \mathrm{~mm}$ ). Fig. 4(a) shows the camera view with the third die located at this position. Fig. 4(b) shows the average of the bounds $\xi_{+}^{\#}, \xi_{-}^{\#}$ versus the $x-z$ position of the third die (specifically, 


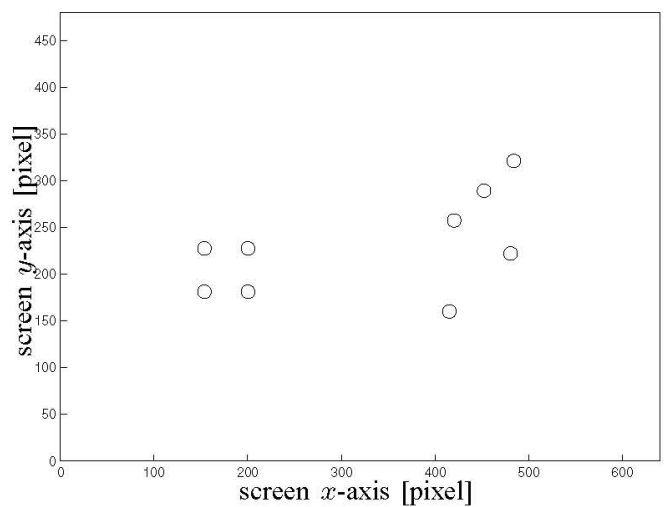

(a)

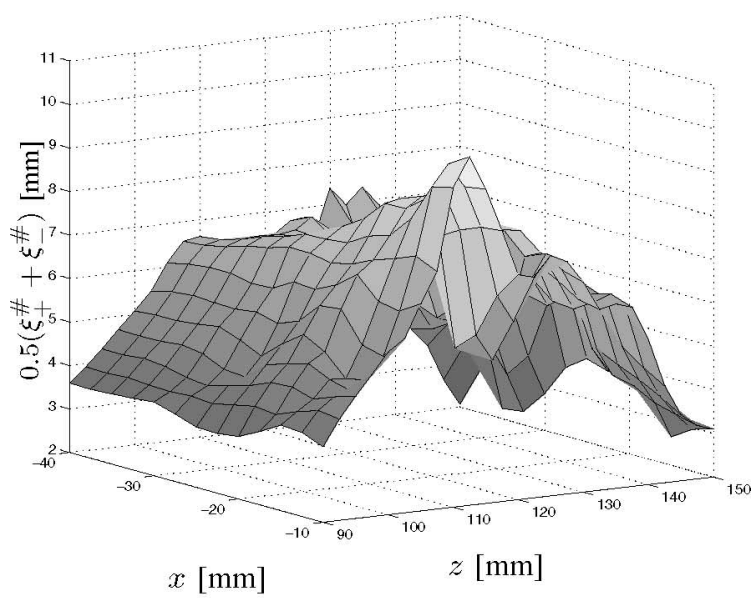

(b)

Fig. 4. Example 2. (a) Camera view with third dice located in the allowed area. (b) Average of the bounds $\xi_{+}^{\#}, \xi_{-}^{\#}$ for the translational error versus the $x-z$ position of the third dice.

this position refers to the center of the die): as we can see, the worstcase positioning error can vary significantly, depending on the position of the dice.

\section{CONCLUSION}

This paper has investigated the positioning error that occurs in visualservoing schemes due to the presence of image noise. In particular, it has been described how upper and lower bounds of the worst-case positioning error for a maximum error of the image points can be computed via optimization problems with LMIs and barrier functions. These bounds are exploited in order to determine optimal object configurations that minimize this error, for instance, in the problem of selecting an optimal subset of object points or determining an optimal position of an object in the scene. As shown by some illustrative examples, the worst-case positioning error can significantly vary, depending on the object configuration.

Future work will investigate the possibility of establishing tightness of the computed bounds, reducing their conservatism, and constructing an overall optimization procedure for determination of optimal object configurations that is entirely convex.

\section{ACKNOWLEDGMENT}

The author thanks the Editors and Reviewers for their valuable comments.

\section{REFERENCES}

[1] G. Chesi, "Camera displacement via constrained minimization of the algebraic error," IEEE Trans. Pattern Anal. Mach. Intell., vol. 31, no. 2, pp. 370-375, Feb. 2009.

[2] G. Chesi, "Estimating the domain of attraction for non-polynomial systems via LMI optimizations," Automatica, vol. 45, no. 6, pp. 1536-1541, 2009.

[3] G. Chesi, "Visual-servoing path-planning via homogeneous forms and LMI optimizations," IEEE Trans. Robot., vol. 25, no. 2, pp. 281-291, Apr. 2009.

[4] G. Chesi, "Time-invariant uncertain systems: A necessary and sufficient condition for stability and instability via HPD-QLFs," Automatica, vol. 46, no. 2, pp. 471-474, 2010.

[5] G. Chesi, A. Garulli, A. Tesi, and A. Vicino, Homogeneous Polynomial Forms for Robustness Analysis of Uncertain Systems. New York: Springer-Verlag, 2009.

[6] G. Chesi and K. Hashimoto, Eds., Visual Servoing Via Advanced Numerical Methods. New York: Springer-Verlag, 2010.

[7] G. Chesi, A. Tesi, A. Vicino, and R. Genesio, "On convexification of some minimum distance problems," presented at the Eur. Control Conf., Karlsruhe, Germany, 1999.

[8] G. Chesi and H. L. Yung, "Performance limitation analysis in visual servo systems: Bounding the location error introduced by image points matching," in Proc. IEEE Int. Conf. Robot. Autom., Kobe, Japan, 2009, pp. 695-700.

[9] P. I. Corke and S. Hutchinson, "A new partitioned approach to imagebased visual servo control," IEEE Trans. Robot. Autom., vol. 17, no. 4, pp. 507-515, Aug. 2001.

[10] N. J. Cowan and D. E. Chang, "Geometric visual servoing," IEEE Trans. Robot., vol. 21, no. 6, pp. 1128-1138, Dec. 2005.

[11] L. Deng, W. J. Wilson, and F. Janabi-Sharifi, "Characteristics of robot visual servoing methods and target model estimation," in Proc. IEEE Int Symp. Intell. Control, Vancouver, BC, Canada, 2002, pp. 684-689.

[12] N. Gans and S. Hutchinson, "Stable visual servoing through hybrid switched-system control," IEEE Trans. Robot., vol. 23, no. 3, pp. 530540, Jun. 2007.

[13] K. Hashimoto, T. Kimoto, T. Ebine, and H. Kimura, "Manipulator control with image-based visual servo," in Proc. IEEE Int. Conf. Robot. Autom., 1991, pp. 2267-2272.

[14] V. Kyrki, "Control uncertainty in image-based visual servoing," in Proc. IEEE Int. Conf. Robot. Autom., Kobe, Japan, 2009, pp. 15161521.

[15] V. Kyrki, D. Kragic, and H. Christensen, "Measurement errors in visual servoing," Robot. Auton. Syst., vol. 54, no. 10, pp. 815-827, 2006.

[16] V. Kyrki, D. Kragic, and H.I. Christensen, "Measurement errors in visual servoing," in Proc. IEEE Int. Conf. Robot. Autom., New Orleans, LA, 2004, pp. 1861-1867.

[17] E. Malis, "Visual servoing invariant to changes in camera-intrinsic parameters," IEEE Trans. Robot. Autom., vol. 20, no. 1, pp. 72-81, Feb 2004.

[18] E. Malis, F. Chaumette, and S. Boudet, "2 1/2 D visual servoing," IEEE Trans. Robot. Autom., vol. 15, no. 2, pp. 238-250, Apr. 1999.

[19] G. Morel, P. Zanne, and F. Plestan, "Robust visual servoing: Bounding the task function tracking errors," IEEE Trans. Control Syst. Technol., vol. 13, no. 6, pp. 998-1009, Nov. 2005.

[20] R. M. Murray, Z. Li, and S. S. Sastry, A Mathematical Introduction to Robotic Manipulation. Boca Raton, FL: CRC, 1994.

[21] B. Thuilot, P. Martinet, L. Cordesses, and J. Gallice, "Position based visual servoing: Keeping the object in the field of vision," in Proc. IEEE Int. Conf. Robot. Autom., Washington, DC, 2002, pp. 1624-1629. 